\title{
Pictures at an Exhibition: Design of a Hybrid Puppetry Performance Piece
}

\author{
Ali Mazalek ${ }^{1}$, Michael Nitsche ${ }^{1}$, Claudia Rébola ${ }^{2}$, Paul Clifton ${ }^{1}$, \\ Andy $\mathrm{Wu}^{1}$, Nick Poirier ${ }^{1}$, and Firaz Peer ${ }^{1}$ \\ 1 Digital Media, Georgia Institute of Technology, \\ Atlanta, GA 30332, USA \\ \{mazalek, michael.nitsche, party, andywu, nick. poirier, firazpeer\}@gatech.edu \\ 2 Industrial Design, Georgia Institute of Technology, \\ Atlanta, GA 30332, USA \\ crw@gatech.edu
}

\begin{abstract}
Pictures at an Exhibition is a physical/digital puppetry piece that uses tangible interface puppets to modify a virtual scene projected at the back of the stage in real-time. The piece merges traditional puppeteering practices with tangible interaction technologies and virtual environments to create a novel performance for the live stage. This paper describes the design and development of piece, as well as our lessons learned from this process and from on-stage performances of Pictures at an Exhibition in a puppetry theatre.
\end{abstract}

Keywords: physical/digital puppetry, performance, experimental theatre, tangible interaction, virtual space.

\section{Introduction}

Digital technologies are increasingly shaping novel performance practices 34 . Hybrid performance formats are found in traditional theatrical settings, as well as in film and video games. In theatre, digital media are used to augment performances, e.g. in the form multimedia projections, in a manner similar to traditional visual effects. Artists and researchers have also explored digital media in combination with real-time computation in order to create interactive experiences for audiences or performers. In film and television, the use of digital puppetry and motion capture technologies is a widespread production process. More recently, the emerging machinima art form uses real-time game engines as platforms for digital puppetry in the creation of short animated films.

Building on these ideas, our work explores the merger of traditional puppetry with tangible interfaces and virtual environments. In Pictures at an Exhibition, five physical puppets equipped with sensors communicate data about their movements to a virtual scene projected at the back of the stage, which changes in response (see Fig. (1). The piece was inspired by Zbig Rybczynski's Tango, which uses 36 overlaying loops to build a video performance. In order to create Tango, 
Rybczynski had to draw and paint roughly 16,000 cell-mattes and make several hundred thousand exposures on an optical printer, a lengthy process that took seven months [14. Instead of looping the recorded image, our piece captures animation data from puppets during the live performance. This data directly affects the virtual scene but is also looped and layered much like the actions in Tango. However, recording and looping happens in real-time. The virtual space becomes a lasting reflection of the story unfolding in the physical space. The abstract mapping between puppets and virtual scene addresses key questions of digital performance that deal with the relationship of the body to the digital realm in hybrid performance pieces.

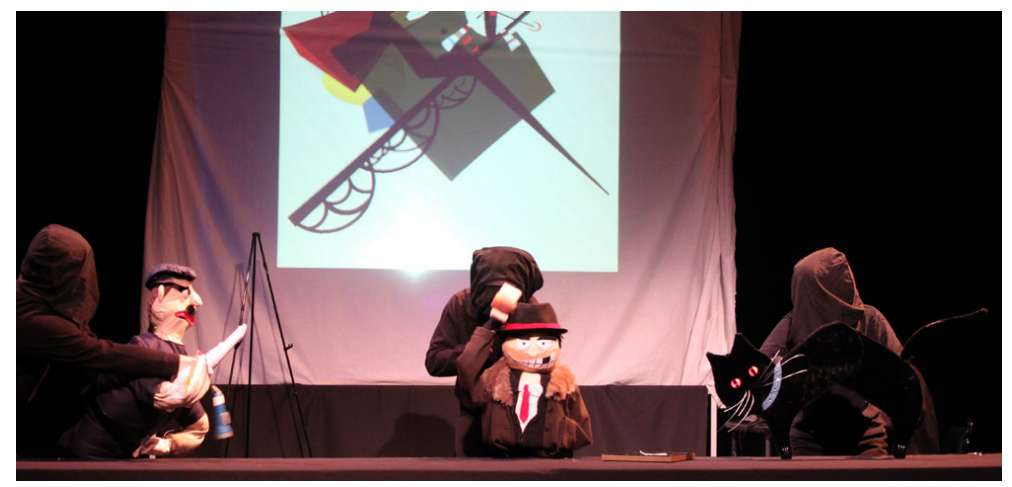

Fig. 1. Performance of Pictures at an Exhibition during the XPT show at the Center for Puppetry Arts

Pictures at an Exhibition was created through a process of physical/digital codesign and was realized as a joint project across classes on experimental media, industrial design, and expressive virtual space. In this context, the conceptual, physical, and digital aspects of the piece co-evolved. The design and development of each aspect-story and performance, physical puppet interfaces, virtual environment-thus informed and altered the design of the other two. The process concluded with the performance of the piece on stage as part of the Xperimental Puppetry Theater show at the Center for Puppetry Arts in Atlanta, GA. The project enabled us to better understand the nature of hybrid performances that bridge the physical and digital worlds, and how to foster them in an interdisciplinary collaboration between artists and researchers.

First, this paper looks at related work from traditional and experimental puppetry. Next, we provide an overview of Pictures at an Exhibition and describe the design process across the physical, digital, and performance aspects of the piece. We discuss experiences and observations from the performances and conclude with lessons learned about the design of hybrid performance pieces for the live stage. 


\section{Related Work}

Our work is inspired and informed by traditional puppetry and by digital technologies in performance arts, especially virtual environments and games. We offer an overview of related work in these areas.

\subsection{Traditional Puppetry}

As we have discussed elsewhere, there is a roughly inversely proportional relationship between ease of use and expressive potential with respect to traditional puppetry techniques [8]. Marionettes, which use strings to control individual parts of the puppet, can lead to a very fine degree of control over the puppet. However, each body part requires an additional string, and each string adds a degree of complexity to the puppet's control. At times, the control is shared. In the Bunraku tradition, three puppeteers control a single puppet. The main puppeteer controls the right arm and the head, while the other two control the left arm of the puppet and its legs. Each puppeteer has a fine degree of control over one or two body parts, which leads to an extremely expressive but also complex performance. Mastering these formats often takes years of practice.

Hand and rod puppets are more limited in their manipulation. However, as Jim Henson's Muppets have shown, in the hands of a skilled puppeteer they can be very expressive. Hand and rod puppets are controlled using one hand inside the puppet and the other hand to move a rod attached to one of the puppet's hands. While hand and rod puppets do not provide a fine degree of control of each individual part of the puppet's body, they do provide a balance between ease of use and expressive range. They can gesture using their arms and can direct their gaze at the important parts of a scene. Certain features, like the eyes, are simplified and exaggerated. Pictures at and Exhibition uses hand and rod puppets designed as described below.

\subsection{Digital Technologies in Theatre Performance}

Pinhanez describes theatre practices that actively use computers during live performance while maintaining the dynamics of live theatrical performance [10]. This includes human-controlled digital lighting and scenery, as well as artificially intelligent actors sharing the stage with a human actor. For example, in Mark Reaney's production The Adding Machine, off-stage computers control 3D projected props and scenery that users at computer terminals move to simulate movement of the live actors on stage. This literally shifts the perspective of the audience. Off-stage actors are also projected in $3 \mathrm{D}$. The size and angle of the projection changes as they interact with the actors performing on the stage [13]. Computation in The Adding Machine is used to frame the viewers' experience in a way that would not be possible otherwise. In comparison, It/I creates a digital character that reacts to the performance of the actor and to audience participation. The digital character communicates with words, pictures, videos, sounds, and stage lighting [11]. 
In puppetry, digital technology has been used primarily for stage effects. Meridiano Theatre's Genesis uses projections to move the scene from underwater to outer space, and projection mapping to highlight certain props with changing surface textures [12. In Terrapin Puppet Theatre's The Falling Room and the Flying Room, a projected television screen acts as a character in the performance. Animated versions of the puppets and actors move back and forth from the animated world to the real world. While the animated events are prerendered, careful staging and skilled puppeteering make the interactions look seamless [16. Digital technologies also allow new forms of audience participation. Naked Puppets' Dark Earth uses technology to enable the audience to affect the scenery, soundscape, and imagery of the performance [9]. In both theatre and puppetry performances, computer vision and projection mapping have been the tools of choice for facilitating interaction between performers, puppets, and digital content. Embedded sensor technology, though, remains undeveloped in live theatre, with few examples of puppets that sense their own movement, like we implemented in Pictures at an Exhibition.

\subsection{Digital Puppetry and Virtual Environments}

Ever since users gained control over interactive digital characters, this interaction has been characterized as a form of dramatic performance [7] and digital puppetry [5]. A player character's actions usually depend completely on a user's input - much like that of a physical puppet. Calvillo-Gamez and Cairns draw a parallel between users learning game controls and puppeteers learning the functionalities of a puppet 2]. But due to the standardized nature of video game systems, the puppet analogy also has deficiencies.

One problem is mapping the limited control schemes of existing interfaces onto the near limitless range of expression. The hardware limits the input to certain set parameters. Keyboard, mouse, joystick, gamepad, motion-control device, etc. each have their own restrictions. In traditional puppetry, each puppet features a control scheme customized for its particular construction. Since games depend on standard input devices, such fine-tuning is difficult. Mappings can be adjusted, as players can set their own control preferences, but remain limited by the hardware. Another challenge is the principle difference in design between games and performances. Video games are defined as goal-driven systems that encourage players to fulfill a certain objective [6]. They emphasize the notion of interaction as making a "choice" 15] that has certain consequences in the game world. Due to the limitations of interaction possible in these worlds, these choices are often simplified (shoot the enemy or not, help the thief or the victim). In contrast, a theatrical performance consists not only of the action a character does, but also how any activity is performed. Due to the dominance of either pre-produced motion captured animations or procedural animation systems such fine-tuned control remains largely inaccessible to users.

Cases of successful digital puppetry grow in emergent play formats, when players either modify or repurpose game elements to achieve a level of expression that was not envisioned by the game designer. For example, fighting moves 
might be re-interpreted as dance for a machinima music video (see Bainst's Dance Voldo Dance). More open systems, like Second Life, allow creation of new animation cycles that can be used by virtual performers like the Ballet Pixelle [1] to stage choreographies with multiple dancers steered in real time by their users. However, their character control is achieved through elaborately timed animation activation. The players have no direct control over individual body parts of their dancing avatars.

Few titles allow for such a direct control over limbs, head, and expression through the interface. Media Molecule's Little Big Planet (2008) features typical jump'n run gameplay but allows players direct control over some facial animations and body parts of the virtual puppet. These expressions have no effect on achieving the main game objective but illustrate the focus on player involvement that is a key component of Little Big Planet. The game includes elaborate creation tools for players to generate game levels and customize their characters. It highlights a shift away from the pre-fabricated goal driven game and exemplifies the expressive range of digital puppetry. As it stands, this shift in commercial video games most often still depends on the inventive use of the underlying platform by the player and is rarely actively supported by the original producer.

\section{$3 \quad$ Pictures at an Exhibition}

Pictures at an Exhibition is a six-minute physical/digital puppetry piece that tells the story of a heist in an art gallery in seven scenes. The cast features five characters: security guard, cat, old lady, small boy, and gangster, each represented by a tangible interface puppet controlled by a single puppeteer. The piece is set in one room of the art gallery, where the old lady, small boy, and gangster conspire to steal a painting (represented on stage by a physical prop), while the security guard and cat try to foil their efforts. Another artwork is projected at the back of the gallery room and acts as a virtual mirror of the characters' actions, reflecting them in abstract animations on its surface, which are looped and layered over time. The piece is a comic reflection on the way the stories of visitors to an exhibition become part of the exhibited paintings themselves. Inspired by the structure of Rybczynski's Tango, the performance unfolds as follows:

- Scene 1: a performer (security guard puppeteer) enters stage and tells a story with their puppet. This puppetry leaves traces on the artwork in the background. Once this section of the story is completed, the performer and puppet leave the stage, but the animations repeat in an endless loop on the artwork at the back of the stage.

- Scene 2: another performer (cat puppeteer) enters and their actions with the puppet animate another part of the virtual canvas. They are soon followed by the first performer (security guard puppeteer), whose new actions are reflected onto the painting and layer with those of cat. As the scene ends, the performers leave the stage but their past actions continue to loop on the virtual canvas. 
- Scenes 3-5: These scenes repeat the pattern, each bringing a different group of characters onto the stage as the story builds up to its climax. At the end of scene 5, the painting prop is stolen and all characters leave the scene while the artwork reflects the frenzy of actions.

- Scenes 6-7: As the story resolves itself with a comic twist involving the whole cast of characters, the layered animations on the painting build up to reveal the fully assembled image as the curtains close.

The story is conveyed through the puppet performance, which is set to a specially composed sound track that uses a different instrument for each character and helps to pace the performance. All puppets are custom-designed variations on hand and rod puppets with sensors at key points of articulation to capture expressive actions. The sensors in each puppet are connected to an Arduino and XBee, which transmit data to a computer that controls the virtual space.

\section{Design Process and Performance}

The piece was designed as part of a joint project across three research and studio classes on experimental media, industrial design, and expressive virtual space at Georgia Tech in spring 2011. One of our goals in producing the piece was to explore, engage in, and ultimately better understand how an interdisciplinary physical/digital co-design process can be applied to the creation of hybrid performance pieces that use real-time computational media on the live stage. Crucial to this is the seamless integration of the physical performance and the digital media on stage. Tangible interaction and puppetry are especially suited for this, since the puppets themselves can serve as an interface between the physical and digital worlds. The performers tell the story through the movements they make with their puppets, while the puppets capture real-time data of these actions and communicate them to the virtual space. The puppets can also serve as reflections of the captured data, for example through LED lights embedded in their bodies.

The project began with an initial concept of the structure and story for the piece. The realization of this concept unfolded across the three classes in a tightly coordinated manner. The progress in each class informed the work in the other two, and also evolved and altered the structure and story to its final state. Students in the three classes worked in teams to develop the different aspects of the piece. In the industrial design course, students did studies of form, materials, and movements, in order to inform the conceptual and physical design of the puppets. In the experimental media course, students explored physical interactions and sensor technologies, in order to realize the design, prototyping, and development of the puppet interfaces. In the expressive virtual space course, students explored the structure and animation potential of visual artworks, in order to create real-time animated versions that could reflect the story of the puppets into the virtual space.

The co-design process required sharing work-in-progress material across all three classes, including design sketches, renderings, technical specifications, and 
prototypes. E.g. an OSC-based communication protocol between the physical interfaces and virtual artwork was developed and refined as the piece evolved. Over the course of the semester, students from each class presented deliverables in the other two classes at key stages in the design process. Subsequent deliverables in those classes built on the ideas and artifacts presented: industrial design students built on the conceptual puppet designs from the experimental media students and presented additional design explorations back. Experimental media students used their output to revise their concepts and develop prototypes of the puppet interfaces. These prototypes were presented to the expressive virtual space students, who in turn used them to inform the design of the virtual artwork.

The Xperimental Puppetry Theatre (XPT) show at the Center for Puppetry Arts in Atlanta accepted our piece. So, in addition to our class collaboration, we also worked with professional puppeteers involved in XPT. These puppeteers provided feedback on the puppet designs, story, and staging of the piece throughout the design process, and helped to coach the performers. One of the puppeteers was a composer and created the music for the piece.

\subsection{Puppets and Story}

The experimental media class was divided into five teams of 3-4 students. Each team was assigned one of the five story characters and tasked with building its corresponding puppet interface. The creation of the puppets and the script evolved in iterations, from concept designs, through two rounds of prototypes, to final products. The two industrial design students worked as a team, producing design concepts and prototypes, which were informed by and inspired the work of the experimental media students.

Phase 1: Concept Designs. Experimental media students first generated conceptual designs for their puppets. The group visited the workshops, stage, and museum at the Center for Puppetry Arts to draw inspiration from traditional puppetry. As they developed their designs, each team was encouraged to consider:

- How their characters personality could be reflected in the puppets physical design and expressive movements;

- How their puppets actions could be captured with sensing technologies and in turn reflected within its body;

- How their characters relationship with other characters could be conveyed through interactions with them; and

- What kinds of story scenarios might unfold between the characters in the art gallery setting.

This conceptual design exercise yielded puppets with a lot of complexity in their mechanical design and operation, and a lot of detail in their physical appearance. The designs supported many different interactions between characters, and thus 


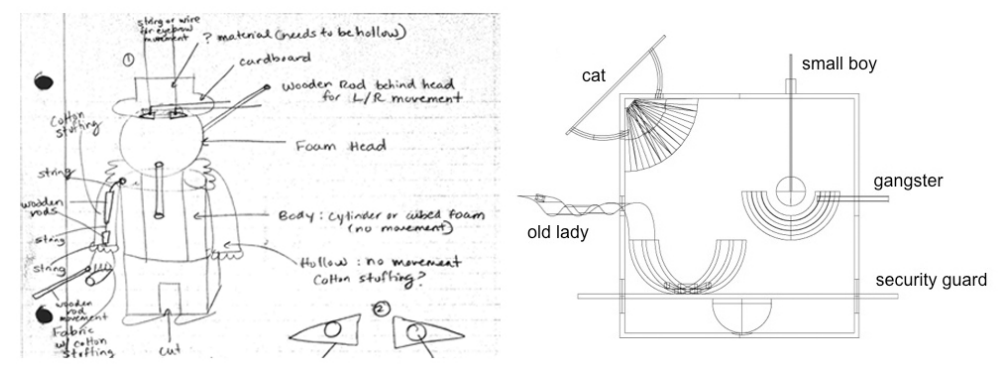

Fig. 2. First design concept for the gangster puppet with complicated control mechanisms (left) compared with the heavily abstracted puppet designs (right)

many possible relationships and story scenarios. The next task was to constrain and significantly simplify these designs.

The industrial design students worked from the initial design concepts, distilling each character's personality to one primary emotion. They explored how these emotions could be conveyed through abstractions of form and movement, and built a cardboard prototype that embodied the five characters as geometric shapes that were integrated into the space of the stage and interacted with one another through simple but evocative movements (see Fig. 21). This prototype was presented to the experimental media students, and encouraged them to radically re-think the nature of character and story in stage performance. Inspired by the work of the industrial designers, the experimental media students then revisited their initial concepts in order to represent each character as a simple geometric shape that communicated its essential qualities: the short stocky gangster was represented by a square, the broad-shouldered security guard was represented by an upside down triangle, the active chubby small boy was represented by a circle, the little old lady in a long dress was represented by a triangle, and the sleek cat was represented by a sideways oval.

Phase 2: Prototyping. Following the initial concept design phase, the experimental media and industrial design students went through two rounds of prototyping. The first prototype focused on the physical design of the puppets, including form, appearance, movement, control, and sensing. Each puppet was designed with sensors (accelerometers, bend sensors, and rotary potentiometers) at key points of articulation, which were connected to an Arduino microcontroller board. Puppets were also equipped with LEDs that highlighted key physical features. E.g., the cat could arch its back and its LED eyes would go from blue to red. The arching movement was detected by two accelerometers placed in the forward and rear segments of the cat's body. The second prototype refined the physical designs, and served as a step in wirelessly communicating puppet data 
to a computer via XBee, where it was visualized using $2 \mathrm{D}$ avatars created in Processing. A standardized XML format was developed to describe the moving parts and data value ranges for each puppet in order to enable calibration and mapping of the sensor data to the virtual artwork running in Unity.
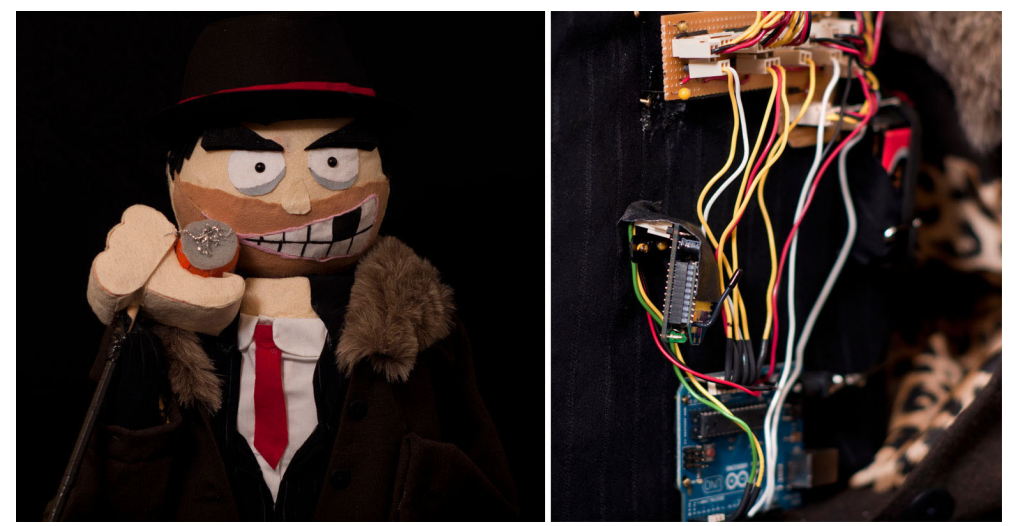

Fig. 3. Final gangster puppet (left) and electronics housed inside the gangster's coat (right). The gangster used a bend sensor to detect movement in his rod-controlled elbow, a force sensing resistor (FSR) to detect contact of his cigar with his mouth, and two 3 -axis accelerometers (one in the head, on in the body) to detect head and body movements.

Phase 3: Final Puppets. Building on the two rounds of prototyping, the industrial design students did studies and design exercises with different materials in order to improve the production quality, consistency, robustness, and performance controls of the final puppets (see Fig. [3). For example, they refined the production of the cat's eyes (using halved ping pong balls) in order make the LEDs more visible on stage, and they added volume to the old lady's dress so that she would better retain her shape while performing.

Story Script. As the puppets and their personalities took shape, so too did the story and dramaturgy. After the first prototypes were built, a story workshop was held with the experimental media students and professional puppeteers. Different story scenarios were storyboarded and eventually turned into a precisely timed script, which was shared with the expressive virtual space students, who used it to refine their designs for the virtual artwork. The script continued to evolve throughout the remainder of the design process, until the puppets, the virtual artwork, and the music were all finalized and the piece was in the final stages of rehearsal. 


\subsection{Virtual Artwork}

The expressive virtual space class was divided into 4 teams of 3-4 students. Their task was to design and animate a virtual environment based on an existing artwork. They remained updated on the development of storyline and characters in the parallel course throughout the process.

Phase 1: Selection and Design. Students had to select an existing artwork to adapt for the virtual canvas. They explored materials and art historical references to envision interaction and animation designs that would reflect its specific qualities. The goal was interactive re-interpretation rather than reproduction. Four reference pieces were selected: French's Summer (1978), Kandisky's On White II (1923), Warhol's Silver Clouds (1966), and van Gogh's Starry Night over the River Rhone (1888); all created in different periods, using different techniques, and engaging audiences in different ways.

For each artwork, the mappings from expressive movements of the puppets to animations on the artwork were done in different ways. Each piece featured some specific quality that was highlighted in the interaction design. E.g., the design for Summer, a virtual stained glass window, referenced glass material in the animations (light reflections, melting, shattering, and bending glass based on external input). On White II, ultimately chosen for the performance, referenced the artist's abstract geometrical art. It does not claim to truly incorporate Kandinsky's complex approach, but uses its distinct components, breaks them into individual 3D objects (see Fig. 四), and maps them onto different characters. The designers looked for features in the painting that would reflect the story characters and their puppets, and the animations follow the lines of movement in the artwork in a way that relates to the key actions made by the puppets. For example, the black cat puppet who leaps and bounds across stage in the performance is reflected in the leaps of the black diagonal segments that cross in the center of the painting, while the gangster smoking his cigar creates bubbles that emerge from the smoke-like curves in the upper left.

Phase 2: Implementation. All four teams created 3D models of their pieces in Maya and imported them into Unity as real time rendered environments. Then they coded the interaction design. Changes in the digital artworks were planned in correlation to the storyline as it evolved from the parallel class.

The most challenging part was connecting the puppet data to the virtual artwork. Seamless communication and clear mappings between the two had to be established. Each puppet had different sensors, which changed throughout the design process. The mapping to animations in the virtual Kandinsky had to cater for these changes. Even during the rehearsal stage, this mapping was adjusted in collaboration with the puppeteers and puppet designers. Unity also had to record the animations in real-time and provide instant playback. This was achieved via a database that recorded all input from the puppets and fed it back into the 

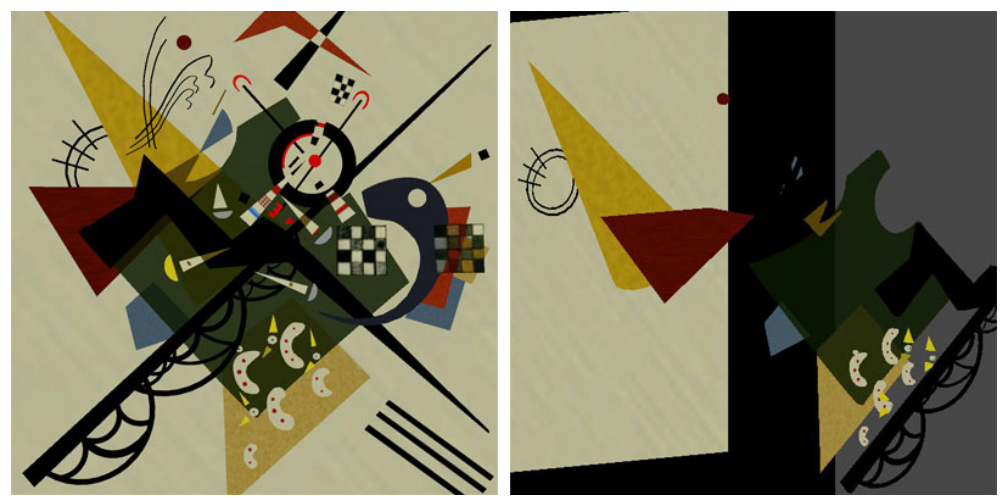

Fig. 4. The virtual artwork - a 3D digital recreation of Kandinsky's On White II. Complete front view (left) and animated portion (right).

render engine when activated by an operator. This system allowed the operator to orchestrate a smaller version of layered animation as seen in Rybczynski's Tango in real-time. We also included pre-set animations that reflected the intended effects. This allowed us to test the artwork while the puppets were still being built. It also served as back-up in case the connection to the puppets was lost.

\subsection{Live Performance}

The XPT show (see Fig. [5] ) consisted of five performances preceded by five nights of dress rehearsals. Overall, the system worked solidly during all rehearsals and dress rehearsals. However, some initially small inconsistencies observed in the the connection between puppets and virtual artwork became more prominent after the first two show performances. This forced us to sidestep the live-recorded animation playback during two of the performances and employ our back-up plan of playing pre-recorded animations on the virtual Kandinsky painting.

Despite the technical difficulties we experienced, the piece received laughter from the audience at the right moments (e.g. when the old lady hit the boy with her cane) and much applause. The feedback received from audience members after each performance was positive, and many people approached us to say how much they enjoyed the piece. Nobody we spoke with had experience of real-time computation used in performances, and many were intrigued by the idea and interested to see more similar works. While everyone perceived the connection between the puppets and the virtual artwork in our piece, relatively few understood how the different characters' stories played out on the virtual canvas. This indicated that the mappings between the puppets' actions on stage and the virtual artwork were too abstract, and would have benefited from simplification. 


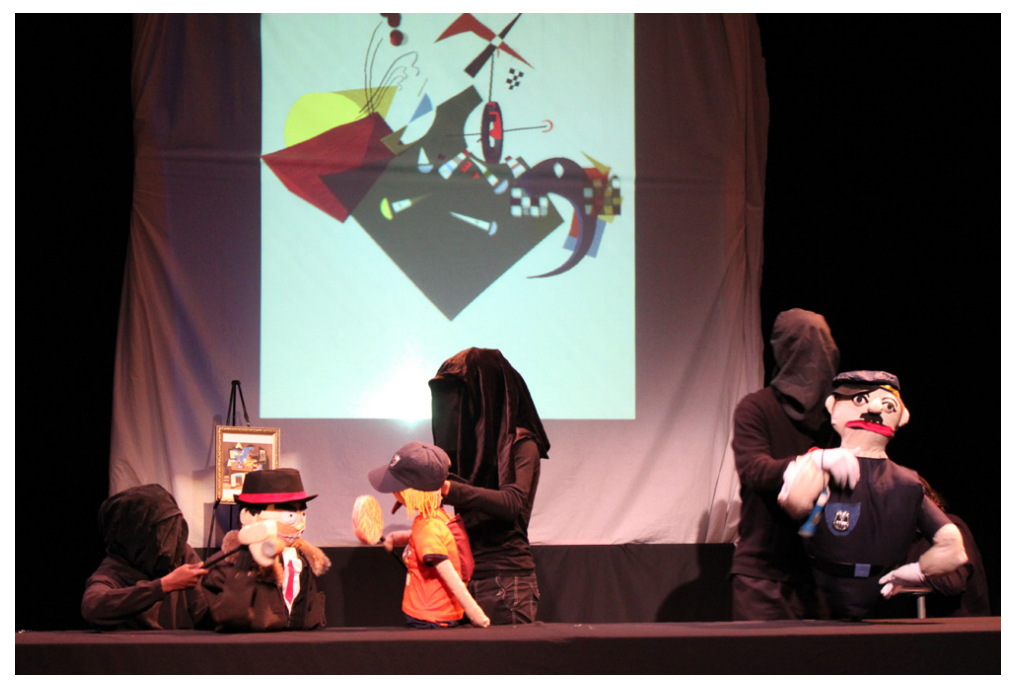

Fig. 5. Performers manipulate the puppets on stage during the XPT performance of Pictures at an Exhibition. The puppets' actions are mapped to the virtual painting in real time.

\section{Discussion and Conclusions}

We provide here a brief summary and discussion of lessons learned from the design and performance of the piece.

Communication. One of the most important aspects of design of a hybrid performance piece is effective and timely communication between members of the interdisciplinary team that is realizing the physical and virtual aspects of the piece. The communication between the industrial design and experimental media students worked well at the first, but became more challenging as the prototypes progressed. Since much of the ideation took place separately, as the designs progressed, students from the two classes were not always in line with their refinements and design iterations. In the same way, communication between experimental media and expressive virtual space students met with challenges throughout the process. Most significantly, the expressive virtual space class would have benefited from having a complete set of captured data earlier in the design process, however this was impossible for the experimental media class to deliver as the piece and technologies were continually evolving. Overall, a more tightly integrated design process in which all three classes participated in all design sessions together would have been beneficial, but this was not feasible due to institutional constraints, such as class scheduling and coordination across different units. 
Mapping. Hybrid performance pieces need to consider how the physical world on stage and the projected virtual world interact. We aimed for a balance between the two. However, the mappings were not obvious enough to the audience, and so the action on stage took precedence over the changes happening to the virtual canvas. In retrospect, this could to some extent have been avoided if we had been able to incorporate the virtual artwork into the rehearsals earlier on. But due to timing constraints and technical challenges, the real-time communication between the puppet interfaces and the artwork was not in place early enough for the rehearsals to happen with the virtual canvas running. A lot of rehearsing/coaching with the professional puppeteers thus focused on the physical performance, and the changes in the artwork did not seem to be designed into the story action from the outset. In the future, it would be worthwhile to explore how to mock up the virtual scene and rehearse with it early in the design process even as the puppet designs, technologies, and storyline continue to evolve.

Balance. Hybrid performances need to consider how to balance between action on the physical stage and action in the virtual space. There is no one correct way to realize this balance since it depends on the story and director's vision. However, it is difficult for the audience to focus in many places at once, so a hybrid piece should give the audience time to take in and switch between action on the physical stage vs. on a projection screen. In our case, replaying animations on the virtual canvas after each scene gave the audience time to process and relate the virtual performance to the physical one. This was especially important given the fast pace of the actions unfolding on stage.

Control. The physical actions made by puppeteers when their focus is on controlling a virtual entity are different than when their focus is generating a physical performance. Our puppeteers performed actions regardless of how they were unfolding on the virtual canvas. Even when communication lagged between the puppets and virtual artwork, the puppeteers maintained their focus on the physical performance. Shifting their focus to control of the virtual, e.g. to make an adjustment or accommodate latency, would have been at the expense of expressivity and narrative communication of the physical puppets. In this sense, a physical performance with a physical controller can be at odds with a virtual performance with a physical controller. This poses a challenge for the creation of hybrid pieces that blend both physical and virtual performances.

Error-Handling. Hybrid performance practices need to develop methods for real-time error handling that do not disrupt the performance in progress. Technological devices and networks rarely work flawlessly. However, common errorhandling methods usually involve pausing the normal workflow in order to address the error (e.g. respond to an error dialog, restart the application, restart the machine, etc.). This is not an option in a live performance, where any disruption will break the fourth wall for the audience. Having a backup plan is important, however the decision to use it needs to be made in a timely manner, 
before the performers are on stage. Solutions enabling the seamless transition to a backup plan in mid performance could be worth exploring in future hybrid performance works.

Acknowledgments. We thank students in our spring 2011 classes at Georgia Tech, puppeteer collaborators Lee Bryan, Dolph Amick, Mauree Culberson and Lynn Talley, XPT director Michael Haverty, the Center for Puppetry Arts, and BlinkM.

\section{References}

1. Ballet Pixelle (2011), http://psg.com/ pixelle/

2. Calvillo-Gamez, E.H., Cairns, P.: Pulling the strings: A theory of puppetry for the gaming experience. In: Guentzel, S., Liebe, M., Mersch, D. (eds.) Proc. of The Philosophy of Computer Games Conference, pp. 308-323. Potsdam University Press (2008)

3. Dixon, S.: Digital Performance. MIT Press, Cambridge (2007)

4. Giannachi, G.: Virtual Theatres: An Introduction. Routledge, London (2004)

5. Hayes-Roth, B., van Gent, R.: Improvisational Puppets, Actors, and Avatars. In: Pahlka, J. (ed.) CGDC Proceedings, pp. 199-209. Miller Freeman, San Francisco (1996)

6. Juul, J.: Half-Real: Video Games between Real Rules and Fictional Worlds. MIT Press, Cambridge (2005)

7. Laurel, B.: Computers as Theatre, 2nd edn. Addison-Wesley Publishing Company, Reading (1993)

8. Mazalek, A., Nitsche, M., Chandrasekharan, S., Welsh, T., Clifton, P., Quitmeyer, A., Peer, F., Kirschner, F.: Recognizing Self in Puppet Controlled Virtual Avatars. In: Proc. Fun and Games 2010, pp. 66-73. ACM (2010)

9. Naked Puppets: Dark Earth. Naked Puppets Theatre Company, Olympia, WA, USA (2006), http://www.nakedpuppets.org

10. Pinhanez, C.S.: Computer Theater. Tech Report 378, Perceptual Computing Group, MIT Media Lab (1996)

11. Pinhanez, C., Bobick, A.: It / I: A Theater Play Featuring an Autonomous Computer Character. Presence: Teleoperators and Virtual Environments 11(5), 536-548 (2002)

12. Ravicchio, G.: Genesis. Meridiano Theatre, Denmark (2006), http://www.meridiano.dk/

13. Reaney, M.: Virtual Scenography: The Actor/Audience/Computer Interface. Theatre Design and Technology 32(1), 36-43 (1996)

14. Rybczynski, Z.: Looking to the Future: Imagining the Truth. In: Penz, F., Thomas, M. (eds.) Cinema \& Architecture: Melies, Mallet-Stevens, Multimedia, pp. 182-198. BFI, London (1997)

15. Salen, K., Zimmerman, E.: Game Design and Meaningful Play. In: Raessens, J., Goldstein, J. (eds.) Handbook of Computer Game Studies, pp. 59-81. MIT Press, Cambridge (2005)

16. Terrapin Puppet Theatre: Hobart, Tasmania, Australia (2011), http://www.terrapin.org.au 